rev Psi

Revista de Psicología (UNLP)

https://revistas.unlp.edu.ar/revpsi

\title{
La multimodalidad en el desarrollo comunicativo temprano
}

\author{
Ana Laguens ${ }^{1}$ \\ Correspondencia \\ analaguens@gmail.com

\section{Filiaciones institucionales} \\ ${ }^{1}$ Centro de Estudios en Nutrición y Desarrollo \\ Infantil (CEREN), Comisión de Investigaciones \\ Científicas de la Provincia de Buenos Aires (CIC) \\ (Argentina) \\ ${ }^{2}$ Universidad Nacional de La Plata, (UNLP, \\ Argentina)
}

\author{
Mariela Resches ${ }^{1}$
}

${ }^{3}$ Centro Interdisciplinario de Investigaciones en Psicología Matemática y Experimental (CIIPME), Consejo Nacional de Investigaciones Científicas y Técnicas (CONICET, Argentina)

\section{Resumen}

Presentamos una revisión narrativa del abordaje multimodal en el estudio del desarrollo comunicativo temprano. Se examinan los antecedentes tradicionales en este campo y se destaca la necesidad de reexaminar la dinámica de las interacciones "preintencionales", ya que en ellas puede observarse cómo se articulan tempranamente diferentes recursos semióticos que componen el entramado multimodal de la comunicación prelingüística. Se proponen tres giros conceptuales para dar cuenta de dicho entramado: la reconsideración del carácter triádico de las primeras interacciones; la función comunicativa y el carácter cultural de los objetos y la resignificación de los gestos ostensivos en tanto signos de pleno derecho. Se analizan los elementos considerados en tanto predictores de la emergencia del lenguaje. Se concluye la necesidad de adoptar una mirada más amplia, que revalorice aspectos habitualmente relegados en el estudio del desarrollo comunicativo temprano y permita formular nuevos interrogantes acerca de sus vínculos con el desarrollo lingüístico inicial.

\section{Palabras clave}

multimodalidad | comunicación prelingüística | interacción social | desarrollo del lenguaje

\section{Cómo citar}

Laguens, A., Querejeta, M. y Resches, M. (2021). La multimodalidad en el desarrollo comunicativo temprano. Revista de Psicología. HTTPS://DX.DOI. ORG/10.24215/2422572XE087

\section{Proceso editorial}

Recibido

12 ago. 2020

Aceptado

11 feb. 2021

Editores

Nicolás Alessandroni, Ana Moreno-Núñez Facultad de Psicología, Universidad Autónoma de Madrid (España)
ISSN

2422-572X

Licencia

Licencia de Cultura Libre CC-BY 4.0

(Compartir - Adaptar - Atribuir)

Entidad editora

RevPsi es una publicación de la

Facultad de Psicología (Universidad

Nacional de La Plata, Argentina) 


\section{Multimodalidade no desenvolvimento comunicativo inicial}

\section{Resumo}

Apresentamos uma revisão narrativa da abordagem multimodal no estudo do desenvolvimento comunicativo precoce. Os antecedentes tradicionais neste campo são examinados e a necessidade de reexaminar a dinâmica das interações "pré-intencionais" é destacada, pois neles é possível observar como diferentes recursos semióticos que compõem a estrutura multimodal da comunicação pré-linguística são articulados desde cedo. Três reviravoltas conceituais são propostas para dar conta desta estrutura: a reconsideração do caráter triádico das primeiras interações; a função comunicativa e o caráter cultural dos objetos e a resignificação dos gestos ostensivos como sinais em seu próprio direito. Os elementos considerados como preditores do surgimento da linguagem são analisados. Conclui-se que é necessário adotar uma visão mais ampla, que revaloriza aspectos geralmente negligenciados no estudo do desenvolvimento comunicativo precoce e nos permite formular novas questões sobre suas ligações com o desenvolvimento lingüístico inicial.

\section{Palavras-chave}

multimodalidade | comunicação pré-linguística | interação social | desenvolvimento da linguagem

\section{Multimodality in early communicative development}

\section{Abstract}

We present a narrative review of the multimodal approach in the study of early communicative development. We examine the traditional antecedents and highlight the need to re-examine the dynamics of "pre-intentional" interactions since they show how different semiotic resources that make up the multimodal framework of pre-linguistic communication are articulated at an early stage. Three conceptual shifts are proposed: the reconsideration of the triadic character of the first interactions; the communicative function and the cultural character of the objects and the resignification of ostensive gestures as signs in their own right. The elements considered as predictors of the emergence of language are analysed. It is concluded that it is necessary to adopt a broader view, which revaluates aspects usually neglected in the study of early communicative development and allows us to formulate new questions about its links with the initial linguistic development.

\section{Keywords}

multimodality | prelinguistic communication | social interaction | language development 
Tradicionalmente, los estudios psicolingüísticos han privilegiado el habla por sobre los componentes vocales y gestuales que intervienen en el lenguaje (Murillo y Belinchón, 2013). Los trabajos que han considerado dichos componentes, los han analizado separadamente: por un lado, han examinado la importancia del uso de gestos en el desarrollo lingüístico posterior (Bates, et al., 1979; Blake, et al, 2005; Butterworth y Morisette, 1996; Camaioni, et al., 1991); por otro, han estudiado dicho proceso desde las vocalizaciones tempranas hacia las producciones verbales convencionales (Karousou, 2003; Vihman, et al., 1985).

En los últimos años, numerosas investigaciones han adoptado un enfoque más amplio, destacando la relevancia de estudiar la coordinación y sincronización de vocalizaciones y gestos en tanto sistema integrado que precede y apuntala la emergencia del primer lenguaje (Alibali, et al., 2000; de Ruiter, 2000; Iverson, 2010; Kendon, 1993; McNeill, 1992; Murillo y Belinchón, 2013; Murillo, et al., 2018). Siguiendo a Rodríguez (2012) “... el gesto se combina con una palabra dentro de un compuesto intermodal que debe concebirse como una unidad de significación y que anticipa las combinaciones frásticas, o cuasi-tales, del período subsiguiente" (p. 47). Es decir, los niños y las niñas aprenden el lenguaje en una matriz de interacción social que supone mucho más que la yuxtaposición de dichos componentes aislados (Vigliocco, et al., 2014). Por el contrario, el desarrollo del lenguaje es concebido como el producto emergente de la interrelación entre diferentes modalidades comunicativas (miradas, gestos, vocalizaciones), que poseen modos de evolución y funcionamiento propio. Podría decirse, por ende, que la multimodalidad constituye una propiedad de la comunicación temprana, al tiempo que una nueva perspectiva para su abordaje. Dicha perspectiva conceptualiza la comunicación prelingüística como un entramado de recursos semióticos que se ponen en marcha en interacción con el entorno social, conformando un sistema dinámico complejo que se autoorganiza como producto de su propio funcionamiento.

Como se señaló anteriormente, buena parte de los estudios que han abordado la comunicación en su carácter multimodal han privilegiado la coordinación gesto-palabra y su relación con la emergencia del primer lenguaje. Dichos estudios encontraron que los gestos y el habla no forman un sistema completamente integrado desde el primer año de vida, sino que requieren de algún tiempo para alinearse entre sí. Por un parte, los trabajos considerados "clásicos", sostienen que la sincronización comienza en la fase de transición entre una y dos palabras (Butcher y Goldin-Meadow, 2000; Capirci, et al., 2005). Sin embargo, estudios más recientes (Esteve-Gibert y Prieto, 2014; Romero, et al., 2017; Murillo, et al., 2018) han encontrado indicios de dicha sincronización ya desde la transición a las primeras palabras. Así, Murillo y colaboradoras (2018) analizaron longitudinalmente la sincronía entre gestos y vocalizaciones en diez niños españoles a los 9, 12, 15 y 18 meses, en situaciones de juego semiestructurado. A diferencia de Butcher y Goldin -Meadow (2000) y Capirci et al. (2005), este trabajo proporcionó evidencia empírica de un cambio significativo en la sincronía entre gestos y vocalizaciones alrededor del primer año de vida, es decir, en el momento en que los niños con desarrollo típico comienzan a producir sus primeras palabras. Para las autoras, estas diferencias de resultados podrían atribuirse a los criterios utilizados 
para definir gesto y habla: mientras que en las investigaciones mencionadas no se contabilizaron los gestos producidos con objetos en las manos (excepto el de mostrar) como así tampoco las vocalizaciones que no fueran palabras o protopalabras, Murillo et al. (2018) consideraron como "gesto" cualquier acción motora dirigida a transmitir un significado a los demás, y como "vocalizaciones" todos los sonidos vocales, diferenciando entre balbuceos y palabras. De este modo, sus resultados sugieren la necesidad de ampliar las perspectivas de análisis de la comunicación prelingüística, comenzando por la reconceptualización de sus componentes, entre los cuales el repertorio gestual, sin duda, desempeña un papel central.

La mayoría de las investigaciones emplea la conceptualización de "gesto" adoptada en los trabajos clásicos de Bates y colaboradores (1979): un comportamiento que tiene la intención de comunicar algo. Los estudios tradicionales sobre el inicio de la comunicación intencional han diferenciado entre gestos deícticos y gestos representacionales (Capirci y Volterra, 2008). Los primeros aparecen entre los 9 y 12 meses y son empleados por el niño para solicitar, mostrar, dar, señalar; se utilizan para llamar la atención del adulto sobre objetos y eventos. El significado del referente solo puede identificarse en el contexto físico en el que tiene lugar la comunicación (Bates, et al., 1975). Los gestos representacionales, entre tanto, surgen entre los 12 y los 15 meses y han sido divididos en convencionales y simbólicos. Los convencionales refieren a rutinas sociales, como por ejemplo saludar o mover la cabeza para afirmar o negar algo. Los simbólicos son movimientos que representan la forma o la función de un referente, como aletear los brazos como si volara o sostener un puño vacío en la oreja como si llamara por teléfono. A estos últimos se los concibe como esquemas de juego simbólico (Caselli, 1990).

Dentro de los gestos deícticos, sin duda el más estudiado ha sido el gesto de señalar o pointing. Siguiendo a Bates y colaboradores (1975), el gesto de señalar constituye un tipo de herramienta social que los niños comienzan a utilizar del mismo modo que las herramientas físicas, es decir, en términos de medios para alcanzar un fin. Desde un punto de vista pragmático, podemos distinguir entre dos tipos de gestos de indicación: a) los protoimperativos, cuando el niño señala para que el adulto le alcance un objeto, es decir, "usa" al adulto como mediador para obtener el objeto; b) los protodeclarativos, cuando el niño señala con la intención de capturar la atención del adulto y compartir su interés hacia un determinado referente. En este caso, es el objeto el que se constituye como mediador entre él y el adulto.

Dado su carácter específicamente humano, así como su rol central en el desarrollo de la comunicación intencional, tanto la naturaleza como la génesis del gesto de indicación ha sido objeto de numerosas controversias.

Uno de los debates más interesantes acerca de la ontogénesis del gesto de indicación puede sintetizarse en dos perspectivas sobre la comunicación prelingüística (Tomasello, Carpenter y Liszkowski, 2007). Una visión más conductual según la cual los bebés señalan para lograr efectos de comportamiento en otros (hacer que "hagan" alguna cosa), de manera tal que no requeriría por parte del niño la comprensión 
del adulto como agente mental, sino, antes bien, como agente causal (Camaioini, 1993; Moore, 1996; Moore y D'Entremont, 2001). Desde esta perspectiva se concibe el gesto como emergente de la interacción social. Así, por ejemplo, a partir de un exhaustivo análisis longitudinal acerca del nacimiento del gesto de indicación en un niño durante su primer año, Carpendale y Carpendale (2010) concluyen que el pointing se desarrolla a partir de la orientación del bebé hacia aspectos de su mundo. Solo posteriormente, dicha orientación es interpretada por los adultos como gesto de señalar, volviéndose de este modo una conducta social.

Una segunda visión, a la que podríamos denominar mentalista (Tomasello et al., 2007) sostiene que, desde el inicio, el gesto de indicación implica algún tipo de atribución de estados mentales por parte del niño (Tomasello, et al., 2005; Liszkowski, 2005, 2006; Tomasello et al., 2007). A diferencia de las perspectivas más conductuales, esta visión coloca las capacidades sociocognitivas para la atención conjunta y la cooperación como fundamento de la interacción social (Butterworth, 2003; Tomasello et al., 2007). En apoyo de esta hipótesis, Tomasello y sus colaboradores desarrollaron una amplia serie de estudios en los cuales exploraron las capacidades de producción y comprensión de gestos en niños prelingüísticos. Liebal, Behne, Carpenter y Tomasello (2007), por ejemplo, analizaron la comprensión del gesto de señalar en bebés de 18 meses en dos situaciones cuasiexperimentales que implicaban la realización de acciones distintas, respectivamente, con dos adultos: juntar juguetes en una canasta y apilar anillos en un poste. Pasado un tiempo, al retomar la interacción con cada uno de ellos, los autores observaron que la interpretación que el niño hacía del significado del pointing en cada caso, dependía de la experiencia recientemente compartida con el adulto. Si bien los adultos señalaron el mismo objeto, los bebés extrajeron diferentes significados sobre la indicación basándose en los marcos de atención conjunta implicados en cada una de las situaciones.

Por su parte, algunas investigaciones recientes han profundizado sobre otras dos cuestiones centrales vinculadas a la aparición del gesto de señalar. En primer lugar, el rol de los gestos ostensivos en tanto precursores semióticos de la indicación (Moreno-Núñez, 2014), y, en segundo lugar, la mediación del señalar "tocando" como transición entre las ostensiones y el señalar propiamente dicho (O'Madagain, el al., 2019). Ambos aspectos serán ampliados en el próximo apartado.

Las diferentes miradas acerca de la comunicación prelingüística comentadas hasta aquí apuntan a la necesidad de profundizar tanto en su desarrollo ontogenético como en su naturaleza multimodal. Para ello, en el apartado siguiente, examinaremos una serie de giros conceptuales que nos permitirán relacionar la dinámica de las primeras interacciones triádicas, las herramientas semióticas utilizadas por adultos y niños y el rol de la interacción social en este desarrollo. 


\section{Tres giros conceptuales en el estudio de la comunicación temprana}

En la última década, un grupo de investigaciones ha comenzado a cuestionar algunos de los principios comúnmente asumidos en el estudio de la comunicación temprana. Tal como veremos en los subapartados siguientes, la reconsideración del carácter triádico de las primeras interacciones junto a la importancia otorgada al estudio de los gestos ostensivos y el uso cultural y comunicativo de los objetos, han venido a conmover la idea de que el nacimiento de la intencionalidad constituye un salto cualitativo en el desarrollo de la comunicación temprana.

Todo parece sugerir que, en este ámbito, como en muchos otros, el desarrollo procede de acuerdo a transiciones graduales antes que a reestructuraciones súbitas (Overton y Molenaar, 2015). Es en este sentido que, en los últimos años, una serie de trabajos desde la perspectiva multimodal, han intentado reestablecer el hilo conductor del desarrollo comunicativo. Se trata de dar cuenta del pasaje desde los primeros intercambios, semióticamente mediados gracias a la guía del adulto, hacia la participación en interacciones en las que el niño asume un papel cada vez más activo al tiempo que se apropia de herramientas semióticas progresivamente más complejas.

\section{De las interacciones diádicas a las interacciones triádicas tempranas}

A partir de la década del 70, numerosos estudios comenzaron a centrar su atención en el desarrollo de la comunicación prelingüística. Dichos estudios han ofrecido diversas explicaciones acerca de las transformaciones que posibilitan, a lo largo del primer año de vida, el tránsito de la comunicación al lenguaje. En esta línea, se ubican los aportes clásicos de autores como Elizabeth Bates y sus colaboradores (Bates et al., 1975; 1979) o Colwyn Trevarthen (1999, 2003), entre otros. Mientras que los trabajos de Bates y su grupo, tomando como referencia la teoría de los actos de habla (Austin, 1962/1982; Searle, 1969/1994) colocan el acento en la intencionalidad del acto comunicativo, los estudios de Trevarthen, desde el ámbito de la psicología, conciben el desarrollo de la comunicación prelingüística en términos de cambios en la organización intersubjetiva. Más allá de sus diferencias de enfoque, ambas propuestas entienden la comunicación prelingüística como un proceso en el que ocurre un salto cualitativo desde las interacciones diádicas, preintencionales, entre bebé y cuidador hacia las interacciones triádicas, intencionales, y en las que se predica acerca de referentes externos. Si antes de los nueve meses el niño era capaz de interactuar alternativamente con objetos o con personas, sobre el último trimestre del primer año, las interacciones en las que participa adoptan la forma de un triángulo referencial compuesto por el niño, un adulto y alguna entidad del mundo externo. En esta dirección se ubican las contribuciones de Michael Tomasello y su grupo (Tomasello, 1999; Tomasello y Carpenter, 2007; Tomasello et al., 2005; 2007) quienes a partir de los años 90 dan un paso más allá al dar cuenta de los fundamentos sociocognitivos de la "Revolución de los 9 meses". Tal como se desarrolló en el apartado precedente en relación con el gesto de indicación, el nacimiento de la comunicación intencional y sus correlatos conductuales dan cuenta de las capacidades del sujeto 
para atender al comportamiento de los adultos en relación con los objetos, pudiendo comprender y considerar a otras personas como agentes intencionales. Una de las primeras evidencias de dicha capacidad subyacente son las habilidades de atención conjunta que los bebés comienzan a mostrar sobre el final del primer año y antes de la emergencia del gesto de indicación (Tomasello, 1999; Tomasello y Carpenter, 2007).

Más recientemente, diversas tradiciones de investigación han destacado la necesidad de recuperar una explicación ontogenética para el tránsito hacia la comunicación intencional, poniendo en cuestión, con diversos matices, esta visión hasta cierto punto "discontinua" de la comunicación prelingüística. Es así como una serie de estudios provenientes del ámbito de la psicología cultural, la teoría de los sistemas dinámicos (Smith y Thelen, 2003) y el enfoque semiótico pragmático del desarrollo (Rodriguez y Moro, 1999) han examinado en detalle la dinámica de las primeras etapas "pre intencionales" del desarrollo comunicativo, arrojando nuevas consideraciones acerca de la mediación cultural presente desde los primeros intercambios bebé-cuidador, las raíces tempranas de la atención conjunta y la emergencia de estructuras triádicas de acción conjunta entre el niño, el cuidador y los objetos desde los primeros meses de vida.

En un estudio transcultural en el que se comparó la dinámica de las interacciones verbales-vocales entre bebés de hasta tres meses y cuidadores en dos contextos culturales, alemanes de clase media frente cameruneses de entorno rural, Keller, Otto, Lamm, Yovsi, y Kärtner (2008) han constatado que la estructura temporal de estos primeros intercambios refleja en cierta forma, modelos culturales de interacción. Si bien los patrones de co-acción y alternancia constituyen dos modos universales de estructurar la interacción, el énfasis en cada uno de ellos difiere entre culturas. Mientras que la alternancia propia de la "toma de turnos" predomina como patrón temporal en las interacciones en el contexto occidental, bebés y cuidadores cameruneses organizan sus interacciones verbales- vocales en forma de co-acción, con una mayor participación de la musicalidad y el contacto corporal. Keller y colaboradores, incluso, especulan con la posibilidad de que estas diferencias tempranas en los modos de estructurar la interacción puedan tener repercusiones en términos de estilos cognitivos de aproximación a la realidad: más analítico en el caso del patrón occidental y más holístico para el no occidental.

Además de las interacciones verbales, el intercambio de miradas se encuentra entre las estrategias comunicativas más tempranas en los primeros intercambios cara a cara entre bebé y cuidador. Junto a su valor comunicativo, la evolución de la dirección de la mirada cobra particular relevancia en tanto refleja el desarrollo de la atención en el niño, desde la atención interpersonal reflejada en la mirada mutua al rostro en el sistema diádico bebé-cuidador, hacia el logro de la atención conjunta, característica de las interacciones triádicas y la referencia compartida.

Aunque la mayor parte de las investigaciones sobre la génesis de la atención en la primera infancia se han centrado en niños mayores de 9 meses, desde la perspectiva de los sistemas dinámicos algunos estudios han analizado cómo evoluciona la dirección de la mirada en la interacción entre niños menores de 9 meses y sus madres. 
Recientemente, Nomikou, Leonardi, Rohlfing y Raczaszek-Leonardi (2016) llevaron adelante un estudio longitudinal cuyo propósito era analizar cómo se coordina la mirada antes del logro de la atención conjunta. Los resultados mostraron que a los tres meses, tanto madre como bebé pasan más de la mitad del tiempo mirándose mutuamente. A los seis meses, en cambio, el interés del bebé en el rostro materno disminuye, al tiempo que se dirige hacia otras regiones del cuerpo y a los objetos circundantes, pudiendo advertirse el significado ostensivo de la mirada del niño, lo cual se vuelve aún más pronunciado a los 8 meses. Desde la perspectiva de los sistemas dinámicos, estos hallazgos proporcionan un posible marco explicativo de la transición desde las interacciones diádicas que, aunque como veremos más adelante, no son exclusivas pero sí predominantes durante el primer semestre de vida, hacia las interacciones triádicas posteriores. Inicialmente, el incremento de los periodos de atención y de las habilidades de memoria en el niño, junto al feedback que ofrece la mirada materna impulsa a los bebés a participar en situaciones de atención mutua durante períodos más prolongados, confiriendo al sistema diádico un estado de relativa estabilidad. Poco después, sin embargo, el creciente interés hacia los objetos circundantes que, debe aclararse, el propio adulto ofrece al niño, lleva al sistema hacia una reorganización que conduce a un nuevo estado de estabilidad: la coordinación de la atención entre el interlocutor y los objetos y finalmente hacia la atención conjunta.

Todo parece indicar, por tanto, que el tránsito desde las interacciones diádicas hacia las triádicas, a lo largo del primer año de vida, resulta difícilmente explicable en términos de saltos cualitativos, sino más bien como proceso gradual que se apoya en múltiples influencias: los marcos culturales de interacción, las capacidades cognitivas del niño y su interrelación con las respuestas contingentes del adulto. Es así como desde una perspectiva gradualista y dinámica del desarrollo se trataría más bien del paso de la predominancia de un modo de interacción a otro en lugar de la sustitución de uno por el otro. Ya desde sus inicios, el sistema interactivo adulto-bebé podría asumir formas tanto diádicas como triádicas, siendo las primeras más estables y prevalentes al inicio, mientras que las segundas adquirirán un grado de mayor estabilidad sobre el segundo semestre del primer año. La pregunta entonces es qué habilidades determinan la preponderancia de una $\mathrm{u}$ otra en distintos momentos del desarrollo. Siguiendo la línea de los trabajos iniciales de Tomasello y colaboradores (2005, 2007), De Schuymer, De Groote, Striano, Stahl, y Roeyers (2011) realizaron un estudio longitudinal donde se compararon la duración y la dirección de la mirada, las vocalizaciones y las acciones motoras frente a dos situaciones experimentales, una de carácter diádico y otra triádico, en niños a los 3, 6 y 9 meses de edad. Sus resultados sugieren que en los dos primeros puntos de medición (3 y 6 meses) las respuestas evaluadas, en tanto expresan las habilidades sociocognitivas de las que dispone el niño, le permiten participar con mayor eficacia de las interacciones dentro de un contexto diádico. Entre tanto, el interés por las interacciones triádicas y la capacidad de operar en ese marco aumenta gradualmente hacia el segundo semestre conforme avanza el desarrollo sociocognitivo.

Si bien las habilidades sociocognitivas del niño constituyen, sin duda, uno de los motores 
del desarrollo comunicativo, éstas solo se expresan en el marco de las interacciones con el adulto y en un determinado contexto cultural. Desde una perspectiva sociocultural, es la respuesta contingente del adulto la que proporciona el andamiaje para conducir al niño más allá de sus posibilidades actuales, permitiendo su participación en situaciones triádicas tempranas, las cuales se ubican propiamente en su zona de desarrollo próximo (Vygotsky, 1978). De este modo, es el adulto quien toma la iniciativa y "presta" al niño su propia intencionalidad comunicativa, al tiempo que le presenta el mundo de los objetos en su dimensión cultural (Alessandroni, el al., 2020; Basilio, 2014; MorenoNúñez, el al., 2017; Rodríguez, el al., 2017; Rodríguez, el al., 2015).

Ahora bien, un examen minucioso de las primeras interacciones triádicas nos obliga a profundizar en el tipo de mediadores semióticos que utilizan niños y adultos en su seno, entre los que destacan los primeros gestos ostensivos y el uso cultural de los objetos en tanto herramientas de comunicación. En los siguientes apartados analizaremos más de cerca las contribuciones que en los últimos años se han realizado desde la perspectiva semiótico pragmática del desarrollo, comenzando por las investigaciones en torno a la pragmática del objeto.

\section{De la pragmática del gesto a la pragmática de los objetos: la materialidad de los intercambios comunicativos}

Tradicionalmente considerados como fuentes de conocimiento físico (Piaget, 1950/1985), o como meros referentes del acto comunicativo, los objetos materiales que participan de las primeras interacciones raramente han sido abordados en su doble condición de objetos de aprendizaje cultural e instrumentos de comunicación. Sin embargo, en la última década asistimos a una tendencia creciente a devolver a dichos objetos su carácter eminentemente cultural y comunicativo, de una manera análoga a otros sistemas semióticos de aparición más tardía, como el lenguaje, la escritura o las representaciones gráficas. Esta perspectiva, conocida como Pragmática del Objeto (Rodríguez et al., 2018; Rodríguez et al., 2015) analiza la emergencia del uso cultural de los objetos, en un proceso que parte desde sus propiedades materiales hacia sus usos simbólicos más abstractos.

La Pragmática del Objeto se inscribe dentro de la denominada perspectiva semiótico- pragmática del desarrollo (Rodriguez y Moro, 1999; Rodriguez, 2006). Dicha perspectiva, si bien hunde sus raíces en los presupuestos básicos de la teoría sociocultural (Vygotsky, 1978), busca superar algunas de sus limitaciones nutriéndose de los aportes de la semiótica de Peirce (Peirce, 1868/1987) aplicada al análisis de las herramientas semióticas preverbales, por un lado, y del abordaje metodológico basado en el análisis microgenético de resolución de tareas propuesto por algunos investigadores de la Escuela de Ginebra (Inhelder et al., 1992), y aplicándolo a las interacciones tempranas, por el otro. Al colocar al signo y su relación con el significado en una posición central incluso antes de la aparición del lenguaje, el enfoque semiótico pragmático de desarrollo busca superar el dualismo desarrollo social-desarrollo cognitivo, propio de la epistemología piagetiana, para proponer una 
epistemología unitaria que recupera el postulado vygotskiano acerca del origen socio interactivo de la cognición en el niño (Rodriguez y Moro, 1999; Rodriguez, 2006).

Rodríguez et al. (2018) proponen que los primeros usos culturales de los objetos que el adulto ofrece al niño se apoyan en sus propiedades rítmico-sonoras, aportándole una estructura en la cual insertar su participación. Si bien algunos autores se han interesado por el papel del ritmo en las interacciones cara a cara (Trevarthen, 2003), su función a propósito del uso de objetos sonoros en el seno de los primeros intercambios niño-adulto-objeto ha sido menos estudiado. El ritmo, junto al sonido de los objetos y la melodía que le imprime el adulto, hace posible que éste establezca con el niño un campo de significados compartidos, dada la cercanía con los usos no convencionales que el propio niño hace de los objetos. Así como dichos usos no convencionales permiten una primera aproximación a las propiedades físicas de los objetos, el ritmo y el sonido, al producirse en espacios de interacción con el adulto, abren la puerta al conocimiento acerca de su uso cultural.

En un intento por dar cuenta de las características de estas primeras interacciones, Moreno-Núñez, Rodríguez y del Olmo (2015) llevaron a cabo un estudio longitudinal en el que observaron la interacción entre seis niños y sus madres a los 2, 4 y 6 meses mientras empleaban un conjunto de anillas sonoras y no sonoras, para incitar distintos tipos de intercambio. Las interacciones fueron categorizadas según las herramientas semióticas empleadas tanto por el adulto como por el niño, agregando los componentes rítmicos, sonoros y melódicos de la acción del adulto y los componentes rítmicos y sonoros de la acción del niño. De esta manera, las categorías empleadas para el análisis de las conductas de los niños refirieron a la atención, el uso de los objetos, los gestos ostensivos autodirigidos y las vocalizaciones. Mientras que en el caso de los adultos se categorizaron los usos del objeto según se tratara de demostraciones (distantes o inmediatas) o gestos ostensivos (simples o rítmicos), y el tipo de lenguaje que acompañaba la acción (verbalizaciones o vocalizaciones). Las autoras encontraron que los componentes rítmicos, sonoros y melódicos relacionados con el uso de los objetos integran un sistema semiótico complejo donde el adulto otorga intencionalidad a la comunicación, mientras que el niño responde de manera contingente. Lo hace prestando atención y respondiendo a la propuesta del adulto de formas cada vez más complejas a medida que avanza en edad (Moreno-Núñez et al., 2015). Resultados similares fueron encontrados por las mismas autoras en otro estudio longitudinal más reciente (Moreno-Núñez et al., 2017) en el que analizaron los primeros gestos utilizados por adultos y niños más pequeños aún, entre los 2 y los 4 meses, a través de una situación triádica configurada en torno a una maraca. Las autoras destacan, en particular, que el carácter rítmico de las acciones en torno a dicho objeto facilita la participación del niño en la interacción. Con el propósito de ahondar en las propiedades rítmico-sonoras de los objetos y su rol en la interacción, Alessandroni, Moreno-Núñez, Rodríguez y Del Olmo (2020) llevaron adelante un estudio de caso en el que analizaron la participación de un niño de 2 meses en una situación donde la madre se comunicaba sobre y a través de la maraca utilizando recursos musicales de creciente complejidad. Entre los recursos 
empleados, se encontraron demostraciones rítmicas sonoras (distales o proximales), el uso de la voz y el movimiento de la pierna del niño. Encontraron componentes musicales pertenecientes a tres dimensiones musicales: estructura rítmico-métrica, organización temporal y otros parámetros musicales, los cuales son los elementos centrales de la musicalidad comunicativa (Malloch y Trevarthen, 2009). Es así que este estudio agrega evidencia al campo de investigación sobre cognición social temprana, incorporando nueva información sobre sus aspectos relacionados con la música en las primeras interacciones.

En definitiva, estos resultados sugieren que los primeros usos culturales de los objetos se "acoplan", en cierta forma, a los usos no convencionales de los que el niño ya es capaz, por lo que podríamos decir que se ubican en su zona de desarrollo potencial (Vygotsky, 1978). Los sonajeros, maracas y otros objetos sonoros tienen un uso funcional que no es diferente al de los esquemas de acción que naturalmente un niño pone en marcha ante la presencia de un objeto, a la manera de lo que Piaget denominó reacción circular secundaria (Piaget, 1936/1969). En consecuencia, al mismo tiempo que exploran su entorno, los niños aprenden a usar los objetos con una función culturalmente establecida, y van haciéndolo con diferente grado de complejidad semiótica. Dicho aprendizaje, no obstante, nunca se produce en solitario.

Ahora bien, ¿Cómo llegan los bebés a conocer el uso de objetos culturalmente más complejos, cuyo uso convencional se encuentra más alejado de sus capacidades de exploración? Rodriguez y Moro (1999) y Moro y Rodriguez (2005) estudiaron longitudinalmente seis niños españoles y seis niños suizos a los 7, 10 y 13 meses de edad. Los observaron en sus hogares interactuando con sus madres y se enfocaron, entre otros aspectos, en cómo evoluciona el uso de dos objetos particularmente propicios para observar este pasaje: un teléfono de juguete y un camión con encastres. A lo largo del período estudiado identificaron tres clases de usos de creciente complejidad: un uso no canónico o no convencional caracterizado por la aplicación a los objetos de esquemas sensoriomotrices de acción, conocidos por el niño; un uso protocanónico o canónico emergente, que supone un intento, aunque incompleto o simplificado, de usar el objeto de modo convencional; y finalmente el uso canónico o funcional. Como era de esperar y, en concordancia con otros estudios previos, a los siete meses ninguno de los bebés consiguió realizar un uso canónico o funcional de los objetos ofrecidos, a pesar de que las estrategias comunicativas utilizadas por los cuidadores pretendían orientar al niño hacia este tipo de uso. A los diez meses los niños comenzaron a ejecutar algunos usos convencionales mientras que a los 13 meses esta tendencia se consolidó de una manera clara. Evidentemente, y como también admiten las autoras, esta progresión es paralela a la capacidad del niño para comprender la intencionalidad del acto comunicativo del interlocutor (Rodriguez y Moro, 1999; Dimitrova y Moro, 2013). Sin embargo, a diferencia de otras explicaciones de inspiración sociocognitiva, desde la perspectiva de la pragmática del objeto la evolución desde los usos preconvencionales hacia los usos convencionales de los objetos se despliega gracias al ajuste progresivo, por parte del adulto, de la cantidad y complejidad de los gestos que produce al grado de conocimiento que 
infiere compartido con el niño (Dimitrova y Moro, 2013). En otros términos, es la atribución conocimiento compartido por parte del cuidador antes que la atribución de intencionalidad compartida por parte del niño el factor que lo conduce hacia el uso funcional de los objetos. No obstante, y como discutiremos brevemente en las conclusiones, es probable que a lo largo del desarrollo estos mecanismos actúen en conjunción, teniendo un rol diferente a lo largo del proceso que conduce a la construcción del uso cultural de los objetos por parte del niño. En un interesante paralelismo con la noción piagetiana de permanencia del objeto (Piaget, 1950/1985), cuando los niños llegan a usar los objetos en forma canónica, esto sugiere que han adquirido un tipo de permanencia que no es física sino funcional, compartida con la comunidad (Rodríguez et al., 2018). Dicha permanencia funcional, podríamos decir, se constituye en una suerte de invariante conceptual que hace posible trascender el aquí y ahora a través de nuevos usos simbólicos y creativos de los objetos.

La evolución del uso cultural de los objetos ha concitado la atención de otras perspectivas de investigación como la teoría de los sistemas dinámicos. Es así que, en un estudio longitudinal, Rossmanith, Costal, Reichelt, Lópezy Reddy(2014)intentaron comprender cómo progresa esta capacidad. Para ello analizaron las interacciones espontáneas entre bebés y cuidadores desde los 3 hasta los 12 meses. Observaron que desde los 3 meses de edad niños y adultos participaban en actividades de "lectura" conjunta de libros. Estas primeras interacciones se producían de una manera altamente coordinada: el adulto modelaba el uso del objeto para el niño en espacios de interacción multimodal ajustados a sus posibilidades, mientras éste participaba activamente coordinando su atención entre el libro y el cuidador. El análisis pormenorizado que llevan adelante estos autores nos permiten observar que el desarrollo de las interacciones triádicas sigue una trayectoria no lineal: un primer período de interacción triádica particularmente intensa entre los 4 y 6 meses de edad, seguido de una fase de exploración solitaria (que coincide con el incremento de las habilidades manipulativas por parte del niño), para finalmente resurgir con nuevas cualidades como actividad conjunta a partir de los 9 meses. Es en este punto donde los bebés son ya capaces de utilizar sus capacidades de manipulación en el marco de una actividad socialmente compartida, cuyo significado cultural ha venido construyéndose desde mucho antes de lo habitualmente observado (Rossmanith et al, 2014).

Son escasos los estudios que analizan cómo los niños, partiendo de su conocimiento acerca del uso convencional de los objetos, construyen sus primeros símbolos en situaciones triádicas. Palacios y Rodríguez (2014) observaron longitudinalmente seis díadas madre-niño en una situación de juego libre con los mismos objetos entre los 9 y los 15 meses de edad. Identificaron cinco niveles de uso simbólico, desde el juego funcional, que supone una distancia mínima entre símbolo y referente, pasando por la sustitución de un objeto por otro, hasta llegar, finalmente, a la evocación del uso del objeto en el vacío, sin asistencia material. Mientras que a los 9 meses los niños no eran capaces de realizar usos simbólicos espontáneos, aunque sí de atender y participar de los usos simbólicos propuestos por el adulto, a los 12 meses comenzaron a ejecutar acciones simbólicas con los objetos, principalmente acciones canónicas con 
un sentido lúdico (nivel 1). Tres meses más tarde, sin embargo, los niños observados evidenciaron usos simbólicos de diversos niveles de complejidad, al tiempo que los adultos redujeron este tipo de acciones. Los resultados de este estudio indican que la producción simbólica por parte del niño supone un progresivo proceso de distanciamiento del uso convencional de los objetos. Sin embargo, no se trata de una construcción solitaria, sino que, por el contrario, los adultos apoyan activamente estos primeros usos simbólicos a través de estrategias diversas, que evolucionan de manera acorde al nivel de desarrollo del niño.

\section{De lo distal a lo proximal: los gestos ostensivos y otras herramientas semióticas tempranas}

Hemos visto cómo el análisis de las interacciones triádicas antes de los nueve meses permitió ampliar el horizonte de investigación más allá de la comunicación intencional, para ocuparnos de las características específicas de los mediadores semióticos que caracterizan las estructuras interactivas preintencionales. En el apartado anterior hemos analizado el uso de los objetos en tanto herramientas de comunicación presentes desde el inicio del desarrollo comunicativo. No obstante, tal como lo demuestran los estudios anteriormente reseñados, dicho uso es inseparable del entramado multimodal que forma junto con la mirada, los gestos y vocalizaciones. En este apartado, intentaremos resituar el rol de los gestos ostensivos como precursores del gesto de indicación y otros gestos distales.

Como se ha mencionado anteriormente, el pointing o gesto de indicación ha sido el centro de la mayor parte de las investigaciones en este ámbito (Tomasello, et al., 2007). Sin embargo, tal como sostiene Moreno-Nuñez (2014) la complejidad semiótica del gesto de señalar ("comunicarse con alguien sobre algo, de manera distal") requiere para su desarrollo haber adquirido previamente comportamientos que impliquen referencia compartida en situaciones comunicativas básicas, con sistemas semióticos menos complejos que involucren al objeto de modo proximal.

En este sentido, nuevas investigaciones han analizado en mayor detalle el surgimiento de otros gestos de aparición más temprana, como los gestos ostensivos de mostrar y dar, que ubican su emergencia entre los 8 y 9 meses de edad del niño (Cameron Faulkner, et al., 2015; Rodríguez et al., 2015).

Rodríguez y colaboradoras (2015) sugieren que el desarrollo de la comprensión y producción de los gestos ostensivos es previo a la emergencia del gesto de señalar. Argumentan, en contraposición a otros desarrollos teóricos (Cyrulnik, 2002; Liszkowski, et al., 2007), que los gestos ostensivos constituyen la primera forma de referencia compartida: los niños los producen con intención comunicativa alrededor de los nueve meses de edad (Dimitrova y Moro, 2013; Reddy, 2008), mientras que los primeros gestos indicativos se producen alrededor de los 12 meses (Butterworth, 2003). Por su parte, es en el marco de las primeras interacciones triádicas, cuando el objeto es presentado al niño a corta distancia, que éste puede comprender los gestos ostensivos producidos por los adultos (Moreno-Núñez, et al., 2015). 
Asimismo, desde un enfoque neovygotskiano, y a diferencia de lo señalado en otras investigaciones en la misma línea (Tomasello, 2007; Tomasello, et al., 2007) estudios más recientes como el de Cameron Faulkner et al. (2015) apoyan la idea de una continuidad entre los gestos ostensivos y el gesto de señalar. Dichos autores llevaron adelante un estudio longitudinal con niños entre 10 y 12 meses en el cual analizaron la frecuencia de uso de los gestos de mostrar y dar, y su relación con la aparición posterior del gesto de indicación. Para ello, evaluaron las conductas comunicativas de los bebés en dos situaciones diferentes de interacción con sus madres: una situación que elicitaba el gesto de señalar y otra, los gestos de dar y mostrar. Sus resultados reflejaron que los gestos ostensivos (dar y mostrar) a los 10 y 11 meses se relacionaban con la frecuencia del gesto de indicación a los 12 meses, de manera tal que los primeros podrían considerarse precursores de los segundos.

Algunos estudios muy recientes destacan nuevos aspectos de la emergencia y funciones de los gestos ostensivos (Moreno-Núñez, et al., 2020; Guevara, et al., 2020). Moreno-Núñez, Rodríguez y Zapata (2020) analizaron la producción gestual de seis niños observados entre los 9 y 13 meses en situaciones de juego con sus madres con distintos objetos y juguetes. Los investigadores categorizaron los gestos de acuerdo a su tipo (ostensivos, ostensivo indexicales o indexicales), a su dirección (hacia sí mismo hacia otro) y a su función (exploratoria-privada para gestos autodirigidos e imperativa, declarativa o fática (Phatic) para gestos comunicativos). Los resultados revelaron algunas cuestiones especialmente relevantes acerca de los gestos ostensivos $\mathrm{y}$ sus funciones. Por una parte, entre los 9 y los 13 meses los niños produjeron muchos más gestos ostensivos que mixtos e indexicales. Por otra, se identificaron tanto gestos dirigidos a otros como a sí mismo, de una forma que sugiere una dimensión no solo comunicativa sino reguladora del gesto ostensivo. Las autoras concluyen así, que el repertorio gestual infantil presenta una variedad de funciones que van más allá de las imperativas y declarativas: una función exploratoria-privada en el caso de los gestos dirigidos a sí mismo, y una función que denominan fática en el gesto de dar dirigido a otro. Esta última, propia de los gestos ostensivos, podría concebirse como precursora de la función declarativa del gesto de indicación. No obstante, se necesitan nuevas investigaciones en apoyo de dicha conjetura.

En una dirección similar, Guevara, Moreno Llanos y Rodríguez (2020) abordaron el desarrollo de los primeros gestos y las acciones educativas de los maestros frente a ellos en una clase de jardín maternal durante el desarrollo de tres tipos de actividades: la bienvenida, la hora de la comida y la actividad central de clase. Entre los gestos observados, como en el estudio anterior, destacan aquellos que las autoras conciben como dirigidos hacia sí mismos, principalmente gestos ostensivos con una función autorreguladora.

Como puede observarse a partir de los estudios precedentes, el desarrollo gestual posee dos características destacables: por una parte, evoluciona desde lo proximal a lo distal y por otra, comporta tanto funciones comunicativas (dirigido a los demás) como autorreguladoras (autodirigido). En otros términos, desde una perspectiva 
vygotskiana, se trata de recuperar la doble función de las herramientas semióticas preverbales, de una manera análoga a lo destacado por Vygotsky al referirse al lenguaje como herramienta de comunicación y de pensamiento.

Nuevas evidencias apoyan la dirección proximal-distal en el desarrollo gestual, lo que podría sugerir la continuidad desde el gesto ostensivo (proximal) hacia el gesto de indicación (distal), pasando por un estadio intermedio que supondría la producción de gestos ostensivos indexicales, el señalar tocando. En efecto, a través de tres estudios transversales de corte experimental en el que participaron niños de 18 meses, 3 y 6 años y adultos, O'Madagain, Kachel y Strickland (2019) aportan evidencia en apoyo a la hipótesis según la cual el gesto de señalar procedería de la ritualización de la exploración de los objetos a través del tocar. Dicha hipótesis se sostiene en dos razones: por una parte, los niños exploran los objetos a través del tacto desde los 6 meses de edad como una forma prototípica de señalar "tocando" y por la otra, la frecuencia del toque exploratorio disminuye a partir de los 9 meses de edad, cuando aumenta la frecuencia de los gestos de señalar, lo que podría sugerir que el señalar "toma el control" del tocar. Los hallazgos mostraron que niños y adultos al señalar, no orientan sus dedos como si intentaran crear una "flecha" que se dirige e indica el objeto, sino como si tuvieran el objetivo de tocarlo, girando sus muñecas coincidentemente con la orientación derecha o izquierda de este. Asimismo, advirtieron que, si bien los adultos y los niños mayores son capaces de interpretar los gestos de señalar como flechas, en los más pequeños, la interpretación dominante de un gesto de señalar es como si se tratara de un intento de tocar un objeto. Para los autores, la acción originalmente diseñada para permitir que el bebé explore un objeto con los dedos se convierte en un gesto que sirve para coordinar la atención del lactante y del adulto sobre un objeto, emergiendo de este modo la indicación.

En lo que respecta a la función autorreguladora de los gestos ostensivos, tal como se ha observado en los estudios precedentes (Moreno Nuñez et al. 2020; Guevara et al. 2020), vale la pena mencionar el hecho de que en el mismo período en que los niños producen sus primeros gestos ostensivos para comunicarse, también producen gestos inmediatos que se interpretan como "dirigidos a sí mismos" y cuya función parece ser explorar una parte del objeto para dominar su uso (Moreno-Núñez et al., 2017). Si bien se trata de una categoría que requeriría de una definición operacional más precisa, resulta sugerente la conjetura según la cual el gesto rebasaría su función comunicativa para pasar a ser un instrumento de autorregulación. Desde esta nueva perspectiva, podría considerarse que la autorregulación comienza a operar antes de la aparición del lenguaje, en un sentido que resulta ciertamente novedoso dentro de los enfoques tradicionales de la Psicología Cultural (Vygotsky, 1978). Si esto fuese así, la producción de gestos privados y el uso funcional de objetos sobre el final del primer año, podrían constituirse en subproductos de la interacción social a partir de los cuales apreciar el incipiente desarrollo de las funciones ejecutivas (Zelazo y Müller, 2004). 


\section{Multimodalidad y adquisición del lenguaje revisitados}

Diversas investigaciones han estudiado el rol de la coordinación de componentes gestuales y vocales en el desarrollo del lenguaje. Estos trabajos coinciden en que las combinaciones de vocalizaciones y gestos predicen futuros logros lingüísticos. Por ejemplo, Murillo y Belinchón (2012) observaron que el uso coordinado de gestos (específicamente el pointing), vocalizaciones y mirada social a los 12 meses de edad es un fuerte predictor del desarrollo léxico tres meses más tarde. En el mismo sentido, Wu y Gros-Louis (2014) e Igualada, Bosch y Prieto (2015) confirmaron que las coordinaciones de gesto y vocalizaciones a los 12 meses se relacionaban con las habilidades lingüísticas a los 15 y a los 18 meses, respectivamente. Parece, por tanto, que la coordinación de elementos gestuales y vocales tiene un papel facilitador en las primeras etapas del desarrollo del lenguaje, posiblemente relacionado con la respuesta diferencial que proporcionan los cuidadores a los intentos comunicativos multimodales de los infantes (Fasolo y D'Odorico, 2012).

Murillo y colaboradores (2018), en el estudio longitudinal ya mencionado, observaron diez niños cada tres meses desde los 9 hasta los 18 meses de edad. Hallaron que los bebés de 12 meses con puntuaciones más altas en comprensión de palabras en el Inventario de Desarrollo Comunicativo MacArthur-Bates (López-Ornat et al., 2005), produjeron con mayor frecuencia coordinaciones entre vocalizaciones y gestos a los 15 meses, lo que a su vez correlacionó con su producción de palabras tres meses después. Asimismo, encontraron que los niños con vocabularios receptivos limitados a los 12 meses produjeron comportamientos comunicativos multimodales menos sincrónicos a los 18 meses. Para las autoras esto podría sugerir que los niños con un mayor vocabulario receptivo a los 12 meses eran capaces de sincronizar gestos y vocalizaciones para transmitir un significado a los demás. Estas habilidades se traducirían en mayores oportunidades para aprender el lenguaje y, por ende, se reflejarían en un vocabulario expresivo más amplio a los 18 meses. Aunque se trata de datos correlacionales con una muestra pequeña, lo cual obliga a ser cautelosos respecto de las conclusiones, todo parece sugerir que la sincronía multimodal puede ser un índice de desarrollo del lenguaje en los comienzos del aprendizaje de palabras.

En una dirección similar a los trabajos anteriormente reseñados, Donelland, Bannard, Mc Gillion, Slocombe y Matthews (2019) intentaron identificar qué componentes de la comunicación prelingüística observados al año de edad, constituían los mejores predictores del desarrollo del vocabulario expresivo un año más tarde. Observaron, en primer lugar, que si bien a los 11 meses los bebés coordinaban de una manera sistemática gestos, vocalizaciones y miradas, cuando la mirada se dirigía hacia el rostro del cuidador incrementaban significativamente las posibilidades de respuesta por parte de éste. En segundo lugar, y en relación con ello, encontraron que el compuesto intermodal vocalización-mirada (antes que el gesto), junto con la respuesta contingente del cuidador, fueron los mejores predictores del tamaño del léxico expresivo a los 24 meses. Estos resultados, por una parte, enfatizan la importancia de analizar la comunicación intermodal en los contextos interactivos 
en los que tiene lugar. Por otra parte, si bien a los 12 meses las vocalizaciones intencionales y claramente dirigidas al interlocutor parecen tener un rol de mayor relevancia predictiva que los gestos, estos hallazgos no contradicen otros previos, sino que es probable que los gestos se constituyan en predictores más importantes del desarrollo lingüístico en un momento posterior, a lo largo del segundo año de vida (Rowe y Goldin-Meadow, 2009). Cabe la posibilidad, por tanto, que las relaciones entre multimodalidad y desarrollo lingüístico temprano sean de naturaleza dinámica e incluso bidireccional, por lo cual se requiere de una mayor cantidad de estudios longitudinales que clarifiquen el sentido de estas relaciones.

\section{Conclusiones}

En este manuscrito hemos examinado diversos aspectos de la comunicación prelingüística que defienden la necesidad de su abordaje desde una perspectiva multimodal. Para ello, hemos apelado a evidencia empírica procedente principalmente de tres tradiciones de investigación acerca de la comunicación temprana: por una parte, la perspectiva semiótica pragmática (Rodriguez y Moro, 1999) y la aproximación sociocognitiva, neovygotskiana, del desarrollo comunicativo (Tomasello, 2020), ambas enraizadas en el marco más amplio de la teoría sociocultural del desarrollo. Por otra parte, también hemos tomado en consideración algunos aportes relevantes de la teoría de los sistemas dinámicos (Smith y Thelen, 2003), cuyos antecedentes pueden remitirse a los modelos neopiagetianos que ponen en valor la última etapa, más funcionalista, de la obra de Piaget.

Los mencionados enfoques presentan diferencias en torno a ciertas tensiones que atraviesan las explicaciones sobre el desarrollo: continuidad vs. discontinuidad del proceso evolutivo, el peso de la herencia biológica en relación a la herencia cultural y las relaciones entre comunicación y cognición. Un análisis a fondo de estas cuestiones excedería los límites de este trabajo. No obstante, a modo de ejemplo, podríamos citar el debate acerca de la intencionalidad del acto comunicativo. La teoría sociocognitiva explica el tránsito hacia la comunicación intencional como una "revolución" que introduce un elemento discontinuo en el desarrollo: la emergencia de la intencionalidad compartida como una capacidad cognitiva específicamente humana, de orden biológico, que solo se pone marcha si existen condiciones individuales y contextuales que la posibiliten (Tomasello, 2007). Por su parte, la perspectiva de la pragmática del objeto asume una posición más continuista, al sostener que esta misma capacidad para la comunicación intencional surgiría como producto de la interacción social, en una dirección de "afuera-adentro" que implica el traspaso de la atribución intencional que en un inicio está a cargo del adulto hasta que el niño toma a su cargo dicha capacidad a través del proceso de internalización.

Los estudios que hemos revisado a lo largo de este trabajo nos sugieren que las antinomias señaladas podrían ser hasta cierto punto aparentes. Como asegura el propio Tomasello en la introducción a Los orígenes culturales de la cognición humana: “...cualquier investigación seria de la cognición humana debe incluir una explicación 
de los procesos históricos y ontogenéticos posibilitados pero no determinados por la adaptación biológica de los seres humanos a una forma especial de cognición social" (Tomasello, 2007, p. 23). Esto supone que son las explicaciones ontogenéticas basadas en la dinámica de las interacciones tempranas, las que continúan siendo decisivas para dar cuenta de los productos y procesos cognitivos y comunicativos específicamente humanos. Proponer la existencia de una o varias capacidades propias de la especie que establecen ciertas condiciones de inicio para el desarrollo, no significa nada más que retomar lo que Vygotsky (1978) definió como línea natural del desarrollo y sobre la cual no profundizó. Dicha línea natural, a la cual Tomasello ha redefinido como "individual" y que en sus propias palabras otorgan el carácter neovygotskiano a su programa de investigación (Tomasello, 2020), se entrelaza de manera compleja con la línea cultural del desarrollo, a la cual la pragmática del objeto ha hecho contribuciones sin duda novedosas y enriquecedoras. Ambas líneas resultan necesarias, pero no suficientes por sí solas para dar lugar al desarrollo ontogenético, por lo que, desde nuestro punto de vista, también sería interesante abandonar la vieja antinomia entre competencia y actuación. Un "bebé competente" no sería sólo aquel que cuenta con conocimientos biológicamente determinados para comprender su mundo, sino con ciertas condiciones iniciales que solo pueden desplegarse en interacción con un entorno cultural complejo.

Esta misma relación de interdependencia podría trasladarse al marco de los microprocesos interactivos de orden local que configuran el desarrollo comunicativo y de los que da cuenta la teoría de los sistemas dinámicos (Smith y Thelen, 2003). Tal como argumentan Nomikou et al. (2016) al explicar la dinámica subyacente al intercambio de miradas madre-niño, las capacidades cognitivas y comunicativas interactúan y se determinan mutuamente. Por una parte, el desarrollo de ciertos procesos cognitivos básicos (v.g. atención sostenida), atribuibles a aspectos madurativos del sistema nervioso central, modifica el sistema interactivo madrebebé, al tiempo que el feedback materno estimula el desarrollo de dichos procesos en una dirección (v.g. atención conjunta) que no podría concebirse fuera del contexto de la interacción humana.

De este modo, del análisis de las investigaciones reseñadas, surge la necesidad de adoptar una mirada más amplia, que permita superar las tensiones mencionadas otorgando a la comunicación temprana su carácter complejo y multideterminado. Un primer paso en esta dirección, consiste en revalorizar ciertos aspectos frecuentemente relegados en el estudio de la comunicación prelingüística, cuestión que hemos intentado reanalizar bajo la forma de tres "giros conceptuales": la reconsideración del carácter triádico de las primeras interacciones; la inclusión de los objetos como herramientas semióticas de diverso nivel de complejidad y, la puesta en valor de los gestos ostensivos y su papel en el camino hacia la emergencia de los gestos distales.

En conclusión, el análisis de la perspectiva multimodal en el estudio dela comunicación temprana nos proporciona nuevas herramientas teóricas y metodológicas para abordar su relación con la emergencia del lenguaje. Se destaca particularmente la 
necesidad de defender el estudio del lenguaje temprano desde un enfoque multimodal, longitudinal y microgenético, que restablezca su continuidad con las herramientas semióticas que lo preceden. Esto nos permitiría formular algunas preguntas de cara al futuro: ¿existen vínculos directos entre la calidad de las primeras interacciones triádicas y la emergencia temprana del lenguaje? ¿Dicha relación está necesariamente mediada por los elementos propios de la comunicación intencional? ¿Existe relación entre los recursos semióticos que utilizan los cuidadores para presentar los objetos al niño y los que usan en los intercambios vocales verbales? ¿Existe una influencia diferencial entre los componentes de la comunicación prelingüística analizados en este trabajo y la comprensión y producción del primer vocabulario, respectivamente? ¿Cómo cambian estas relaciones a lo largo del desarrollo?

Estos y otros interrogantes constituyen un interesante desafío para el diseño de nuevas investigaciones que permitan, a su vez, optimizar el desarrollo comunicativo infantil a través de estrategias de intervención que tomen en consideración la estructura multimodal de las interacciones tempranas.

\section{Referencias}

Alessandroni, N., Moreno-Núñez, A., Rodríguez, C., y Del Olmo, M. J. (2020). Musical dynamics in early triadic interactions: A case study. Psychological Research, 84(6), 1555-1571. HTTPS://DOI.ORG/10.1007/S00426-019-01168-4

Alibali, M. W., Kita, S., y Young, A. J. (2000) Gesture and the process of speech production: We think, therefore we gesture. Language and Cognitive Processes, 15(6), 593-613. HTTPs://DOI. ORG/10.1080/016909600750040571

Austin, J. L. (1962/1982). Cómo hacer cosas con palabras. Palabras y acciones (G. R. Carrió y E. A. Rabossi, trads.). Paidós.

Basilio Seyler, M. (2014). Los signos preverbales como herramientas de pensamiento: el origen social de la autorregulación cognitiva en niños de 14 a 18 meses de edad. Tesis doctoral inédita. Universidad Autónoma de Madrid. HTTP://HDL. HANDLE.NET/10486/664104

Bates, E., Beningni, L., Bretherton, I., Camaioni, L., y Volterra, V. (1979). The emergence of symbols: Cognition and communication in infancy. Academic Press.

Bates, E., Camaioni, L., y Volterra, V. (1975). The acquisition of performatives prior to speech. Merrill-Palmer Quarterly, 21, 205 - 224. нттр:// WWW.JSTOR.ORG/STABLE/23084619
Blake, J., Vitale, G., Osborne, P., y Olshansky, E. (2005). A cross-cultural comparison of communicative gestures in human infants during the transition to language. Gesture, 5(1-2), 201217. HTTPS://DOI.ORG/10.1075/GEST.5.1.14BLA

Butcher, C., y Goldin-Meadow, S. (2000). Gesture and the transition from one- to two-word speech: When hand and mouth come together. En D. McNeill (Ed.), Language and gesture (pp. 235-258). Cambridge University Press.

Butterworth, G. (2003). Pointing is the royal road to language for babies. En S. Kita (Ed.), Pointing: Where language, culture, and cognition meet (pp. 9 - 33). Erlbaum.

Butterworth, G., y Morissette, P. (1996). Onset of pointing and the acquisition of language in infancy. Journal of Reproductive and Infant Psychology, 14(3), 219-231. HTTPs://DOI. ORG/10.1080/02646839608404519

Camaioni, 1., Caselli, M. C., Longobardi, E. y Volterra, V. (1991). A parent report instrument for early language assessment. First Language, 11(33), 345-358. HTTPS://DOI. ORG/10.1177/014272379101103303 
Cameron-Faulkner, T., Theakston, A., Lieven, E., y Tomasello, M. (2015). The relationship between infant holdout and gives and pointing. Infancy, 20(5), 576-586. HTTPS://DOI.ORG/10.1111/ INFA. 12085

Capirci, O., Contaldo, A., Caselli, M. C., y Volterra, V. (2005). From action to language through gesture: A longitudinal perspective. Gesture, 5, 155-177. HTTPS://DOI.ORG/10.1075/GEST.5.1.12CAP

Capirci, O., y Volterra, V. (2008). Gesture and speech: The emergence and development of a strong and changing partnership. Gesture, 8(1), 22-44. HTTPS://DOI.ORG/10.1075/GEST.8.1.04CAP

Carpendale, J. I. M., y Carpendale, A. B. (2010) The development of pointing: From personal directedness to interpersonal direction. Human Development, 53(3), 110-126. HTTPS://DOI. ORG/10.1159/000315168

Caselli, M. C. (1990). Communicative gesture and first words. En V. Volterra y C. J. Erting (Eds.), From gesture to language in hearing and deaf children (pp. 56-67). Springer Verlag.

Cyrulnik, B. (2002). De la conscience émergente à la conscience partagée. En F. Rastier y S. Bouquet (Eds.), Une introduction aux sciences de la culture (pp.81-90). PUF.

De Ruiter, J. P. (2000). The production of gesture and speech. En D. McNeill (Ed.), Language and gesture (pp. 284-311). Cambridge University Press.

De Schumer, L., De Groote, I., Striano, T., Stahl, D. y Roeyers, H. (2011). Dyadic and triadic skills in preterm and fullterm infants: A longitudinal study in the first year. Infant Behavior and Development, 34(1), 179-188. HTTPS://DOI. ORG/10.1016/J.INFBEH.2010.12.007

Dimitrova, N., y Moro, C. (2013). Common ground on object use associates with caregivers' gestures. Infant Behavior and Development, 36, 618-626. HTTPS://DOI.ORG/10.1016/J.INFBEH.2013.06.006

Donnellan, E., Bannard, C., McGillion, M. L., Slocombe, K. E., y Matthews, D. (2020). Infants' intentionally communicative vocalizations elicit responses from caregivers and are the best predictors of the transition to language: A longitudinal investigation of infants' vocalizations, gestures and word production. Developmental Science, 23(1), e12843. нтTP:// DOI.OR/ 10.1111/DESC.12843

Esteve-Gibert, N., y Prieto, P. (2014). Infants temporally coordinate gesture-speech combinations before they produce their first words. Speech Communication, 57, 301-316. HTTPS://DOI.ORG/10.1016/J.SPECOM.2013.06.006
Fasolo, M., y D’Odorico, L. (2012). Gesture-plusword combinations, transitional forms, and language development. Gesture, 12, 1-15. HTTPS://DOI.ORG/10.1075/GEST.12.1.01FAS

Guevara, I., Moreno-Llanos, I., y Rodríguez, C. (2020). The emergence of gestures in the first year of life in the Infant School classroom. European Journal of Psychology of Education, 35, 265-287. HTTPS://DOI.ORG/10.1007/S10212-019-00444-6

Igualada, A., Bosch, L., y Prieto, P. (2015). Language development at 18 months is related to multimodal communicative strategies at 12 months. Infant Behavior and Development, 39, 42-52. HTTPS:// DOI.ORG/10.1016/J.INFBEH.2015.02.004

Inhelder, B. (1992). Le cheminement des découvertes de l'enfant. (recherche sur les microgenèses cognitives). Delachaux y Niestle.

Iverson, J. (2010). Developing language in a developing body: The relationship between motor development and language development Journal of Child Language, 37(2), 229-261. HTTPs://DOI. ORG/10.1017/So305000909990432

Karousou, A. (2003). Análisis de las vocalizaciones tempranas: Su patrón evolutivo y su función determinante en la emergencia de la palabra. Tesis doctoral inédita. Universidad Complutense de Madrid.

Keller, H., Otto, H., Lamm, B., Yovsi, R. D., y Kärtner, J. (2008). The timing of verbal/ vocal communications between mothers and their infants: A longitudinal cross-cultural comparison. Infant Behavior and Development, 31(2), 217-226. HTTPS://DOI.ORG/10.1016/J. INFBEH.2007.10.001

Kendon, A. (1993). Human gesture. En K. R. Gibson, y T. Ingold (Eds.), Tools, language and cognition in human evolution (pp. 43-62). Cambridge University Press.

Liebal, K., Behne, T., Carpenter, M., y Tomasello, M. (2007). Infants use shared experience to interpret pointing gestures. Developmental Science, 12(2), 264-271. HTTPS://DOI.ORG/10.1111/I.14677687.2008.00758.x

Liszkowski, U. (2005). Human twelve-montholds point co-operatively to share interest with and provide information for a communicative partner. Gesture, 5, 135-154. HTTPS://DOI. ORG/10.1075/GEST.5.1.11LIS

Liszkowski, U. (2006). Infant pointing at twelve months: Communicative goals, motives, and social-cognitive abilities. En N. Enfield y S. Levinson (Eds.), The roots of human sociality: Culture, cognition, and interaction (pp. 153 178). Berg. 
Liszkowski, U., Carpenter, M., y Tomasello, M. (2007). Reference and attitude in infant pointing. Journal of Child Language, 34, 1-20.

López-Ornat, S., Gallego, C., Gallo, P., Karousou, A., Mariscal, S., y Martínez, M. (2005). Inventarios de desarrollo comunicativo MacArthur. TEA Ediciones.

Malloch, S., y Trevarthen, C. (2009). Communicative musicality. Oxford University Press.

McNeill, D. (1992). Hand and mind: What gestures reveal about thought. University of Chicago Press.

Moore, C. (1996). Theories of mind in infancy. British Journal of Developmental Psychology, 14, 19-40. HTTPS://DOI.ORG/10.1111//.2044835X.1996.TB00691.X

Moore, C., y D'Entremont, B. (2001). Developmenta changes in pointing as a function of parent's attentional focus. Journal of Cognition and Development, 2, 109-129. HTTPs://DOI. ORG/10.1207/S15327647JCD0202

Moreno-Núñez, A. (2014). Ostensive gestures in triadic interactions: From rhythmic ostensive gestures of the adult to children's gestures at the end of the first year of life. Tesis doctoral inédita. Universidad Autónoma de Madrid. HTTPS://REPOSITORIO.UAM.ES/BITSTREAM/ HANDLE/10486/664115/MORENO NUNEZ ANA. PDF?SEQUENCE $=1$

Moreno-Núñez, A., Rodríguez, C. y Del Olmo, M.J. (2015). The rhythmic, sonorous and melodic components of adult-child-object interactions between 2 and 6 months old. Integr. Psych. Behav., 49, 737-756. HTTPs://DOI.ORG/10.1007/ $\underline{\text { S12124-015-9298-2 }}$

Moreno-Núñez, A., Rodríguez, C., y Del Olmo, M. J. (2017). Rhythmic ostensive gestures: How adults facilitate infants' entrance into early triadic interactions. Infant Behavior and Development, 49, 168-181. HTTPS://DOI.ORG/10.1016/J. INFBEH.2017.09.003

Moreno-Núñez, A., Rodriguez, C., y MirandaZapata, E. (2020). Getting away from the point: the emergence of ostensive gestures and their functions. Journal of Child Language, 47(3), 556-578. HTTPS://DOI.ORG/10.1017/ S0305000919000606

Moro, C., Rodríguez, C. (2005). L'objet et la construction de son usage chez le bébé: une approche sémiotique du développement préverbal. Peter Lang.
Murillo, E., y Belinchón, M. (2012). Gesturalvocal coordination: Longitudinal changes and predictive value on early lexical development. Gesture, 12, 16-39. HTTPS://DOI.ORG/10.1075/ GEST.12.1.02MUR

Murillo, E., y Belinchón, M. (2013). Patrones comunicativos multimodales en la transición a las primeras palabras: Cambios en la coordinación de gestos y vocalizaciones. Infancia y Aprendizaje, 36, 473-487. HTTPS:// DOI.ORG/10.1174/021037013808200258

Murillo, E., Ortega, C., Otones, A., Rujas, I., y Casla, M. (2018). Changes in the Synchrony of Multimodal Communication in Early Language Development. Journal of Speech Language and Hearing Research, 61(1), 1-11. HTTPS://DOI. ORG/10.1044/2018 ISLHR-L-17-0402

Nomikou, I., Leonardi, G., Rohlfing, K. J., y Rączaszek-Leonardi, J. (2016). Constructing interaction: the development of gaze dynamics. Infant and Child Development, 25(3), 277-295. HTTPS://DOI.ORG/10.1002/ICD.1975

O’Madagain, C., Kachel, G., y Strickland, B. (2019). The origin of pointing: Evidence for the touch hypothesis. Science Advances, 5(7), eaav2558. HTTP://DOI.OR/10.1126/SCIADV.AAV2558

Overton, W. F., y Molenaar, P. C. (2015). Concepts, theory, and method in developmental science: A view of the issues. En R.M. Lerner (Eds.), Handbook of child psychology and developmental science (pp. 1-8) Wiley.

Palacios, P., y Rodríguez, C. (2014). The development of symbolic uses of objects in infants in a triadic context: A pragmatic and semiotic perspective. Infant and Child Development, 24(1), 23-43. HTTP://DX.DOI.ORG/10.1002/ICD.1873

Peirce, C. S. (1868 -1987) Obra lógico-semiótica (R. Alcalde y M. Prelooker, trads.). Taurus.

Piaget, J. (1936/1969). El desarrollo de la inteligencia en el niño (L. Fernández Cancela, trad.) Aguilar.

Piaget, J. (1950/1985). La toma de conciencia (L. Fernández Alfonso, trad.) Ediciones Morata.

Reddy, V. (2008). How infants know minds. Harvard University Press.

Rodríguez, C. (2006). Del ritmo al símbolo. Los signos en el nacimiento de la inteligencia. Horsori.

Rodríguez, C., Basilio, M., Cárdenas, K., Cavalcante, S., Moreno-Núńez, A., Palacios, P., y Yuste, N. (2018). Object pragmatics: Culture and communication - the bases for early cognitive development. En A. Rosa y J. Valsiner (Eds.), The Cambridge handbook of sociocultural psychology (pp. 223-244). Cambridge University Press. 
Rodríguez, C., Benassi, J., Estrada, L., y Alessandroni, N. (2017). Early social interactions with people and objects. En A. Slater y G. Bremner (Eds.), An introduction to developmental psychology (3ra edn., pp. 213-258). Hoboken/West Sussex.

Rodríguez, C., Moreno-Núñez, A., Basilio, M., y Sosa, N. (2015). Ostensive gestures come first: their role in the beginning of shared reference. Cognitive Development, 36, 142-149. https://doi. org/10.1016/j.cogdev.2015.09.005

Rodríguez, C., y Moro, C. (1999). El mágico número tres. Cuando los niños aún no hablan. Paidós.

Rodríguez, F. (2012). El hermano menor de la palabra. Panorámica de los estudios sobre el gesto. Psiencia. Revista Latinoamericana de Ciencia Psicológica, 4(1), 43-56. HTTP://DX.DOI. ORG/10.5872/PSIENCIA.V4I1.88

Romero, A., Etxebarria, A., de Pablo, I., y Romero, A. (2017). Interrelación entre gestos y vocalizaciones en funciones comunicativas tempranas: Evidencias desde la lengua. Revista Signos, 50(93), 96-123. HTTP://DX.DOI.ORG/10.4067/ So718-09342017000100005

Rossmanith, N., Costall, A., Reichelt, A. F., López, B., y Reddy, V. (2014). Jointly structuring triadic spaces of meaning and action: book sharing from 3 months on. Frontiers in Psychology, 5, 1390. HTTPS://DOI.ORG/10.3389/FPSYG.2014.01390

Rowe, M. L., y Goldin-Meadow, S. (2009). Differences in early gesture explain SES disparities in child vocabulary size at school entry. Science, 323(5916), 951-953. HTTPS://DOI.ORG/10.1126/ SCIENCE.1167025

Searle, J. R. (1969/1994). Actos de habla (L.M Valdés Villanueva, trad.). Cátedra.

Smith, L. B., y Thelen, E. (2003). Development as a dynamic system. Trends in Cognitive Sciences, 7(8), 343-348. HTTPS://DOI.ORG/10.1016/S13646613(03)00156-6

Tomasello, M. (1999). The human adaptation for culture. Annual Review of Anthropology, 28(1), 509-529. HTTPS://DOI.ORG/10.1146/ANNUREV. ANTHRO.28.1.509

Tomasello, M. (2007). Los orígenes culturales de la cognición humana (A. Negrotto, trad.) Amorrortu.

Tomasello, M. (2020, 28 de diciembre). Becoming human: a theory of ontogeny [presentación en congreso]. IV Congreso Latinoamericano para el Avance de la Ciencia Psicológica. Disponible en HTTPs://WWW.YoutuBE.COM/ WATCH? $=08$ PBGENETQU\& $\mathrm{T}=158 \mathrm{~S}$
Tomasello, M., y Carpenter, M. (2007). Shared intentionality. Developmental Science, 10(1), 121-125. HTTPS://DOI.ORG/10.1111/J.1467$7687.2007 .00573 . \mathrm{X}$

Tomasello, M., Carpenter, M., Call, J., Behne, T. y Moll, H. (2005). Understanding and sharing intentions: The origins of cultural cognition. Behavioral and Brain Sciences, 28, 675-735. HTTPS://DOI.ORG/10.1017/So140525X05000129

Tomasello, M., Carpenter, M. y Liszkowski, U. (2007). A new look at infant pointing. Child Development, 78(3), 705-722. HTTPS://DOI. ORG/10.1111/J.1467-8624.2007.01025.X

Trevarthen, C. (1999) Musicality and the intrinsic motive pulse: evidence from human psychobiology and infant communication. Musicae Scientiae, 3(1), 155-215. HTTPS://DOI. ORG/10.1177/10298649000030S109

Trevarthen, C. (2003). Conversations with a twomonth-old. Whurr Publishers.

Vigliocco, G., Perniss, P. y Vinson, D. (2014). Language as a multimodal phenomenon: Implications for language learning, processing and evolution. Philosophical Transactions of the Royal Society B: Biological Sciences, 369(1651), 20130292. HTTPS://DOI.ORG/10.1098/ RSTB.2013.0292

Vihman, M. M., Macken, M. A., Simmons, H., y Miller, J. (1985). From babbling to speech: A reassessment of the continuity issue. Language, 61(2), 397-445. HTTPS://DOI.ORG/10.2307/414151

Vygotsky, L. S. (1978/2000). El desarrollo de los procesos psicológicos superiores (S. Furió, trad.). Crítica.

Wu, Z., y Gros-Louis, J. (2014). Infants' prelinguistic communicative acts and maternal responses: Relations to linguistic development. First Language, 34, 72-90. https://doi. org/10.1177/0142723714521925

Zelazo, P. D., y Müller, U. (2004). Executive function in typical and atypical development. En U. Goswami (Ed.), Blackwell handbook of childhood cognitive development (pp. 445-469). Blackwell 